\title{
Laryngeal mask airway insertion by classic and thumb
}

\section{insertion technique: a comparison [version 1; peer review: 2}

\section{approved]}

\author{
Monica Goyal ${ }^{1}$, Akanksha Dutt², Anjum S Khan Joad³ \\ ${ }^{1}$ Department of Anesthesiology, O.P. Jindal Hospital, Hisar, 125005, India \\ ${ }^{2}$ Department of Anesthesiology, Sawai ManSingh Medical College, Jaipur, 302004, India \\ ${ }^{3}$ Department of Anesthesiology, Bhagawan Mahavir Medical Research Center, Jaipur, 302017, India
}

\begin{tabular}{l}
\hline F1 First published: 09 May 2013, 2:123 \\
https://doi.org/10.12688/f1000research.2-123.v1 \\
Latest published: 09 May 2013, 2:123 \\
https://doi.org/10.12688/f1000research.2-123.v1 \\
\hline
\end{tabular}

\section{Abstract}

We evaluated the efficacy of an alternative technique, for insertion of the silicone laryngeal mask airway (LMA) Classic ${ }^{\mathrm{TM}}$ in 40 American Society of Anesthesiologists grade ASA I and II patients scheduled for elective surgery. In group I (Index Finger group), the LMA was inserted by the classic index finger technique and, in group $T$ (Thumb Insertion group), the thumb insertion technique was used. Ease of insertion, fiberoptic laryngoscopic position, cuff pressures and laryngopharyngeal morbidity were assessed in both study groups. On statistical analysis, both groups were comparable in all respects. From our study it can be concluded that thumb insertion is an effective insertion technique for the LMA Classic ${ }^{\mathrm{TM}}$.

Keywords

Laryngeal mask airway, index finger technique, thumb insertion

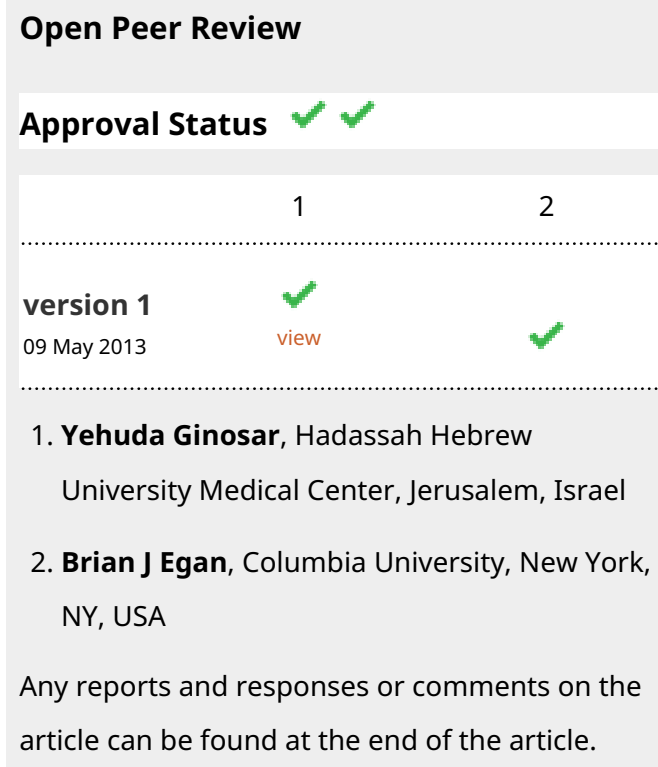

Corresponding author: Akanksha Dutt (akankshadutt@yahoo.co.in)

Competing interests: No relevant competing interests were disclosed.

Grant information: The author(s) declared that no grants were involved in supporting this work.

Copyright: @ 2013 Goyal M et al. This is an open access article distributed under the terms of the Creative Commons Attribution License, which permits unrestricted use, distribution, and reproduction in any medium, provided the original work is properly cited. Data associated with the article are available under the terms of the Creative Commons Zero "No rights reserved" data waiver (CC0 1.0 Public domain dedication).

How to cite this article: Goyal M, Dutt A and Khan Joad AS. Laryngeal mask airway insertion by classic and thumb insertion technique: a comparison [version 1; peer review: 2 approved] F1000Research 2013, 2:123 https://doi.org/10.12688/f1000research.2123.v1

First published: 09 May 2013, 2:123 https://doi.org/10.12688/f1000research.2-123.v1 


\section{Introduction}

The thumb insertion technique offers an attractive alternative technique for insertion of a laryngeal mask airway (LMA). An LMA is conventionally inserted by the index finger insertion technique as described by Dr. Archie Brain ${ }^{1,2}$. However, there are certain conditions where LMA insertion using the index finger is difficult, as it is not possible to reach the head end of the patient, for instance during mass casualty (e.g. fire, building collapse, etc.) or in case of a patient with a stereo tactic frame ${ }^{3}$. Also, paramedical workers are often less comfortable working at the head end of the patient. In such circumstances, an alternative technique of insertion may be useful. Therefore, this study was planned to assess the thumb insertion technique.

This study was performed using the silicone LMA (LMA Classic ${ }^{\mathrm{TM}}$ ) to compare the two insertion techniques with respect to ease of insertion, fiberoptic laryngoscopic position, cuff pressure and patient comfort in patients for elective general anesthesia.

\section{Material and methods}

After approval from the hospital research and ethical committee, a prospective, randomized double-blind study was conducted on patients of American Society of Anesthesiologists ASA grade I \& II between 18-60 years of age, undergoing elective surgery.

Exclusion criteria were as follows ${ }^{1}$ :

1. Risk of aspiration (full stomach, gastroesophageal reflux disease, pregnancy).

2. Mouth opening $<2.5 \mathrm{~cm}$.

3 . Weight $<40 \mathrm{~kg}$ or $>110 \mathrm{~kg}$.

4. Respiratory tract pathology.

5. Cervical spine disease.

After informed consent was obtained, patients were randomly allocated into two groups of 20 each. In group Index (I), the silicone LMA was inserted using the index finger insertion technique and in group Thumb $(\mathrm{T})$, the silicone LMA was inserted using the thumb insertion technique. Randomization was done by opening sealed numbered envelopes.

Patients were premedicated with oral diazepam $0.1 \mathrm{mg} / \mathrm{kg}$ two hours before anesthesia and intravenous Ondansetron $0.01 \mathrm{mg} / \mathrm{kg}$ just before induction of anesthesia. Intra-operative monitoring included continuous electrocardiogram (ECG), non-invasive blood pressure (NIBP), oxygen saturation $\left(\mathrm{SpO}_{2}\right)$ end tidal $\mathrm{CO}_{2}\left(\mathrm{Et} \mathrm{CO}_{2}\right)$ and airway pressure.

Following preoxygenation, anesthesia was induced with intravenous fentanyl $2 \mathrm{mcg} / \mathrm{kg}$. Two minutes after administering fentanyl, propofol $2.5 \mathrm{mg} / \mathrm{kg}$ was given intravenously ${ }^{4}$. One minute after administration of propofol, LMA insertion was attempted. During LMA insertion, if the mouth opening was inadequate, or if purposeful movement in patients was observed, further bolus doses of
$10 \mathrm{mg}$ propofol were given to facilitate insertion. In all patients the LMA was inserted by an experienced anesthesiologist who was well versed with both techniques of LMA insertion.

In group Index (I), the LMA was inserted from the head end of the patient after partially inflating the cuff ${ }^{5}$ (i.e. filled with half the recommended air in the cuff), and lubricating the posterior surface of the cuff with water-soluble jelly. The patient's head was supported on a firm ring with neck flexed and head extended. The tube portion of the laryngeal mask was grasped as if it were a pen; the index finger was pressed on the point where the tube adjoins the mask. The patient's mouth was opened; the tip of the mask was placed against the inner surface of the upper incisors or gums with the aperture facing anteriorly (and the black line facing the patient's upper lip). The mask was pressed back against the hard palate to keep it flattened as it advanced into the hypopharynx, using the index finger to push upward against the palate. The tube was grasped with the other hand, straightened slightly, and then pressed down with a single, quick but gentle movement until a definite resistance was felt.

In group $\mathrm{T}$, the LMA was inserted from the right side of the patient ${ }^{6,7}$, i.e. the operator stood facing the patient, in the angle made by the chest and right arm of the patient. After partially inflating the cuff (i.e. filled with half the recommended air in the cuff), the posterior surface of the cuff was lubricated with water-soluble jelly. The patient's head was supported on a firm ring with neck flexed and head extended. The tube portion of the laryngeal mask was grasped in a pen-like fashion; the thumb (instead of the index finger) was pressed on the point where the tube adjoins the mask. After opening the patient's mouth, the tip of the mask was placed against the inner surface of the upper incisors or gums with the aperture facing anteriorly (and the black line facing the patient's upper incisors). The mask was pressed back against the hard palate to keep it flattened as it advanced into the hypopharynx until a definite resistance was felt. In this technique, the thumb was used to apply pressure against the hard palate while advancing the LMA. The tube was grasped with the other hand while removing the thumb ${ }^{6}$.

After insertion, cuff inflation of either device was to a "just seal" pressure or up to a maximum of $60 \mathrm{~cm} \mathrm{H}_{2} \mathrm{O}$, as measured with a simple hand-held aneroid manometer ${ }^{8}$. The volume of air used was recorded, and a larger device was substituted if leaks persisted on gentle manual ventilation.

Insertion success was assessed by the following criteria:

1. Establishing a clear airway.

2. Rising up of device during cuff inflation.

3. Anterior neck filling with device inflation.

4. The device remained in midline with the black line on the posterior side of airway tube remaining in midline in line with the upper incisors.

Anesthesia was maintained with isoflurane and nitrous oxide in oxygen and vecuronium bromide $(0.08 \mathrm{mg} / \mathrm{kg}$ initially followed by 
$0.01 \mathrm{mg} / \mathrm{kg}$ every 30 minutes). Mechanical ventilation was volume controlled and time cycled with a tidal volume $(5-8 \mathrm{ml} / \mathrm{kg})$ set to maintain peak inspiratory pressure less than $20 \mathrm{~cm}$ of $\mathrm{H}_{2} \mathrm{O}$, and ventilator frequency was adjusted to maintain $\mathrm{EtCO}_{2}$ at $30-38 \mathrm{~mm}$ of $\mathrm{Hg}$ with an I/E ratio of 1:2.

Optimal ventilation was assessed by the following criteria9:
a. Adequate chest expansion.
b. Stable oxygenation.
c. Square wave capnograph.

Soon after this, a fiberoptic bronchoscope was passed through the device and the view was graded as follows ${ }^{10,11}$ :
a. Vocal cords fully visible.
b. Vocal cords \& posterior epiglottis visible.
c. Vocal cords \& anterior epiglottis visible.
d. Vocal cords not seen but ventilation adequate.

For our study, fiberoptic view grades a. and b. were labeled good and $\mathrm{c}$. and d. were labeled poor.

After surgery, neuromuscular block was antagonized with neostigmine $(0.05 \mathrm{mg} / \mathrm{kg})$ and glycopyrrolate $(0.01 \mathrm{mg} / \mathrm{kg})$. LMA was removed after deflating the cuff when the patient regained consciousness and protective airway reflexes. The presence of blood on the LMA cuff was recorded.

In the postoperative period, patients were asked if they had a sore throat in the recovery room and 24 hours postoperatively. The responses were graded as follows.
a. Nil
b. Mild
c. Moderate
d. Severe

Statistical analysis was done using Chi-square test with Yates correction for qualitative analysis and analysis of variance (ANOVA) for quantitative analysis.

\section{Results}

The mean age of first group with index finger insertion was 43.15 years whereas the mean age of second group with index finger insertion technique was 45.70 years. The mean weight of first group was 61.10 and that of second group was 55.3 years. The male: Female ratio in both groups was 1:4. There was no statistically significant difference between the two groups (Table 1). The time taken, number of attempts, cuff pressure and fiberoptic view scores were comparable in the two groups (Table 2). The insertion success of
LMA \& laryngopharyngeal morbidity was statistically comparable between both groups (Table 3). The time taken and number of attempts for LMA classic insertion were comparable irrespective of the technique used. No significant difference was found in the cuff pressure, fiberoptic view scores and pharyngeal morbidity in both study groups. However, the sample size was small.

Results of silicone laryngeal mask airway insertion

3 Data Files

http://dx.doi.org/10.6084/m9.figshare.690946

\section{Discussion}

The manufacturers of the LMA Classic have recommended thumb insertion as one of the methods of insertion ${ }^{7}$, but there is little evidence in the literature available on its success. In this study, an attempt was made to compare the index finger and thumb insertion techniques for the LMA classic insertion. The users of LMA had a shorter learning curve compared to the endotracheal tube, and paramedical workers lacking advanced airway training easily master the skill of inserting an LMA ${ }^{12,13}$. As this group of care givers may not be as comfortable as doctors in working at the head end of the patient, the thumb insertion technique is an attractive option (this technique was demonstrated by Dr. Chandy Varghese at an airway workshop at the All India Institute of Medical Sciences, New Delhi, India in 1999, citing the reluctance of paramedical workers to work at the head end ${ }^{14}$ ). Also, in conditions of mass casualty e.g. fire; building collapse or earthquake, when it may not be possible to reach the head end of the patient, the thumb insertion technique would be useful. The time taken for the successful insertion of an airway device when used for airway management during anesthesia or in apneic patients during resuscitation is crucial and, therefore, has to be reasonably brief. In the present study, the time of LMA insertion was defined as the time taken from picking up the device until the time at which positive pressure ventilation was started. Even though in our study, the mean time taken in the thumb insertion group $(34.00+17.31 \mathrm{sec})$ was longer than in the index finger group $(29.00+28.6 \mathrm{sec})$, the difference was not statistically significant. The number of attempts required for LMA insertion using either technique was also comparable statistically.

Silva and Brimacombe ${ }^{15}$ (1996) described a non-conventional (thumb) insertion technique of the LMA for general anesthesia during stereotactic implantation of fetal hypophysis in Parkinson's disease in five patients, as the conventional approach from the patient's head end was impeded by the stereotactic frame.

The LMA cuff pressures in both study groups were also found to be comparable. A statistically significant difference was not found in the fiberoptic view scores between the two study groups, emphasizing the fact that technique of insertion did not influence correct placement of the LMA and effective ventilation. No significant difference in the incidence of sore throat or blood on device was found in the study groups. 
Table 1. Demographic profile of the patients.

\begin{tabular}{lllll} 
Parameters & \multicolumn{2}{c}{ Group } & P-value & Significance \\
\cline { 2 - 5 } & Index & Thumb & N & \\
Age (years) & $43.15 \pm 7.41 \mathrm{SD}$ & $45.70 \pm 10.86 \mathrm{SD}$ & $>0.05$ & $\mathrm{NS}$ \\
Weight (Kilograms) & $61.10 \pm 17.44 \mathrm{SD}$ & $55.30 \pm 09.55 \mathrm{SD}$ & $>0.05$ & $\mathrm{NS}$ \\
Male: Female & $1: 4$ & $1: 4$ & - & -
\end{tabular}

Both the study groups were statistically similar with respect to age and weight. NS = Not significant.

\section{Table 2. Ease of insertion.}

\begin{tabular}{lllll}
\multirow{2}{*}{ Parameters } & \multicolumn{2}{l}{ Group (Mean \pm SD of Various parameters) } & P-value & Significance \\
\cline { 2 - 5 } & Index & Thumb & $>0.05$ & NS \\
Time taken for insertion (Seconds) & $29.00 \pm 28.60$ & $134.00 \pm 17.31$ & $>0.05$ & NS \\
Cuff pressure $(\mathrm{mm}$ of $\mathrm{Hg})$ & $43.40 \pm 10.79$ & $42.30 \pm 10.53$ & $>0.05$ & $\mathrm{NS}$ \\
No of attempt & $01.20 \pm 000.51$ & $01.25 \pm 00.54$ & $>0.05$ & $\mathrm{NS}$ \\
\hline Fiber optic view & $01.40 \pm 00.58$ & $01.80 \pm 00.81$ &
\end{tabular}

The time taken, number of attempts, cuff pressure and fiberoptic view scores were comparable in the two groups. NS = Not significant

\section{Table 3. Insertion success.}

\begin{tabular}{|c|c|c|c|c|c|c|}
\hline \multirow{2}{*}{ Parameter } & & \multicolumn{2}{|c|}{ Group } & \multirow{2}{*}{ SD } & \multirow{2}{*}{ P-value } & \multirow{2}{*}{ Significance } \\
\hline & & Index & Thumb & & & \\
\hline \multirow{2}{*}{ Clear airway } & Yes & 20 & 20 & - & - & - \\
\hline & No & 0 & 0 & & & \\
\hline \multirow{2}{*}{ Rising up of device } & Yes & 19 & 17 & 0.278 & $>0.05$ & NS \\
\hline & No & 1 & 3 & & & \\
\hline \multirow{2}{*}{ Anterior neck filling } & Yes & 18 & 17 & 0.000 & $>0.05$ & NS \\
\hline & No & 2 & 3 & & & \\
\hline \multirow{2}{*}{ Device remained in midline } & Yes & 20 & 20 & 0.000 & $>0.05$ & NS \\
\hline & No & 0 & 0 & & & \\
\hline \multirow{2}{*}{ Blood } & Present & 2 & 4 & 0.278 & $>0.05$ & NS \\
\hline & Not Present & 18 & 16 & & & \\
\hline
\end{tabular}

The insertion success of LMA \& laryngopharyngeal morbidity was statistically comparable between both groups.

Most of us are accustomed to efficiently placing the LMA by the index finger technique in the operation theatre, but we also need to master the thumb technique to handle difficult situations outside theatre.

\section{Conclusion}

In this patient population, the thumb insertion technique was as effective as index finger insertion technique with respect to ease of insertion and insertion success. It also provides optimal ventilation, comparable fiberoptic view scores and comparable incidence of sore throat. It is important for doctors and paramedical workers to learn both techniques, especially for situations outside the carefully controlled operating theatre environment.

\section{Author contributions}

Anjum and Monica contributed to the conception and design of the study. Monica and Akanksha collected and analyzed the data. Akanksha wrote up the manuscript. Anjum and Akanksha both approved the manuscript.

\section{Competing interests}

No relevant competing interests were disclosed.

\section{Grant information}

The author(s) declared that no grants were involved in supporting this work. 
1. Jerry $A D$, Susan E: Dorsch "Laryngeal mask airways" Chapter $\mathbf{1 5}$ 'Understanding Anesthesia Equipment' 4th Edition. Editor - Sharon Zinner. Published in Pennsylvania, Williams and Wilkins 1999, 477-486.

2. Brain A: Proper technique for insertion of the Laryngeal Mask. Anesthesiology. 1990; 73(5): 1053-4.

PubMed Abstract

3. Silva LC, Brimacombe JR: The Laryngeal mask airway for stereotactic implantation of fetal hypophysis. Anesth Analg. 1996; 82(2): 430-1. PubMed Abstract | Publisher Full Text

4. Goyagi T, Tanaka M, Nishikawa T: Fentanyl decreases propofol requirement for laryngeal mask airway insertion. Acta Anaesthesiol Scand. 2003; 47(6): 771-4. PubMed Abstract | Publisher Full Text

5. Matta BF, Marsh DS, Nevin M: Laryngeal mask airway: A more successful method of insertion. J Clin Anesth. 1995; 7(2): 132-5. PubMed Abstract | Publisher Full Text

6. Khan Rashid M, Bhatti TH, Ahmed Moeid S: Laryngeal mask airway and the difficult airway management. J Anaesth Clin Pharmacol. 2005; 21(3): 241-6. Reference Source

7. updated on $9^{\text {th }}$ September 2003 access accessed on 29th May 2008. LMA North America, Inc. Reference Source

8. Brain Al: Pressure in laryngeal mask airway cuffs. Anaesthesia. 1996; 51(6): 603 PubMed Abstract

9. Shafik MT, Bahlman BU, Hall JE, et al:: A comparison of the Soft Seal disposable and the Classic re-usable laryngeal mask airway. Anaesthesia.
2006: 61(2): 178-81

PubMed Abstract | Publisher Full Text

10. Payne J: The use of the Fibreoptic laryngoscope to confirm the position of the laryngeal mask. Anaesthesia. 1989; 44(10): 865. Publisher Full Text

11. Brimacombe J, Berry A: A proposed fiber-optic scoring system to standardize the assessment of LMA position. Anesth Analg. 1993; 76(2): 457. PubMed Abstract

12. Reinhart DJ, Simmons G: Comparison of placement of the laryngeal mask airway with endotracheal tube by paramedics and respiratory therapists. Ann Emerg Med. 1994; 24(2): 260-3.

PubMed Abstract | Publisher Full Text

13. Deakin CD, Peters R, Tomlinson P, et al:: Securing the pre-hospital airway: A comparison of laryngeal mask insertion and endotracheal intubations by UK paramedics. Emerg Med J. 2005; 22(1): 64-67.

PubMed Abstract | Publisher Full Text | Free Full Text

14. Flaishon R, Sotman A, Friedman A, et al:: Laryngeal mask airway insertion by anesthetists and nonanesthetists wearing unconventional protective gear: a prospective, randomized, crossover study in humans. Anesthesiology. 2004; 100(2): 267-73.

PubMed Abstract | Publisher Full Text

15. Silva LC, Brimacombe JR: The laryngeal mask airway for stereotactic implantation of fetal hypophysis. Anesth Analg. 1996; 82(2): 430-1.

PubMed Abstract | Publisher Full Text 


\section{Open Peer Review}

\section{Current Peer Review Status:}

\section{Version 1}

Reviewer Report 14 May 2013

https://doi.org/10.5256/f1000research.1186.r950

(C) 2013 Egan B. This is an open access peer review report distributed under the terms of the Creative Commons Attribution License, which permits unrestricted use, distribution, and reproduction in any medium, provided the original work is properly cited.

\section{Brian J Egan}

Department of Anesthesiology, Columbia University, New York, NY, USA

Competing Interests: No competing interests were disclosed.

I confirm that I have read this submission and believe that I have an appropriate level of expertise to confirm that it is of an acceptable scientific standard.

Reviewer Report 10 May 2013

https://doi.org/10.5256/f1000research.1186.r939

(C) 2013 Ginosar Y. This is an open access peer review report distributed under the terms of the Creative Commons Attribution License, which permits unrestricted use, distribution, and reproduction in any medium, provided the original work is properly cited.

\section{Yehuda Ginosar}

Department of Anesthesiology and Critical Care Medicine, Hadassah Hebrew University Medical Center, Jerusalem, Israel

This article was well designed, analyzed and presented. It is not "science" but it does address a clinically useful question and does so using an appropriate scientific methodology. The only comments that I would make require minor changes.

I suggest modifying the names of the groups to "anesthesiologist at head of patient" and "anesthesiologist facing patient" - or possibly a more elegant phraseology (south-facing vs north-facing approach?) - this is really the main difference between the techniques whether you use the thumb or index finger is a secondary difference derived from the first. This should also appear in the title.

In table 3, please add a footnote explaining IS1, IS2 etc and OV 1, OV 2 etc - I know these 
terms appear in the text but each table should be self-explanatory.

Competing Interests: No competing interests were disclosed.

I confirm that I have read this submission and believe that I have an appropriate level of expertise to confirm that it is of an acceptable scientific standard.

The benefits of publishing with F1000Research:

- Your article is published within days, with no editorial bias

- You can publish traditional articles, null/negative results, case reports, data notes and more

- The peer review process is transparent and collaborative

- Your article is indexed in PubMed after passing peer review

- Dedicated customer support at every stage

For pre-submission enquiries, contact research@f1000.com 